01

\title{
Математическое моделирование емкостного ВЧ разряда низкого давления, помещенного во внешнее радиальное магнитное поле, посредством программы KARAT
}

\author{
(C) И.И. Задириев, ${ }^{1}$ А.А. Рухадзе, ${ }^{1}$ Е.А. Кралькина, ${ }^{2}$ К.В. Вавилин, ${ }^{2}$ В.Б. Павлов, ${ }^{2}$ В.П. Тараканов ${ }^{3,4}$ \\ ${ }^{1}$ Институт общей фризики РАН им. А.М. Прохорова, \\ 119991 Москва, Россия \\ ${ }^{2}$ Московский государственный университет им. М.В. Ломоносова, \\ 119991 Москва, Россия \\ ${ }^{3}$ Объединенный институт высоких температур РАН, \\ 125412 Москва, Россия \\ ${ }^{4}$ Национальный исследовательский ядерный университет „МИФИ“, \\ 115409 Москва, Россия \\ e-mail: iizadiriev@gmail.com
}

(Поступило в Редакцию 22 апреля 2015 г. В окончательной редакции 23 марта 2016 г.)

Выполнено математическое моделирование высокочастотного емкостного разряда в геометрии плазменного ускорителя с замкнутым дрейфом электронов. Показано, что в подобном разряде, также, как и в разряде постоянного тока, реализуется азимутальный дрейф электронов, и образуется скачок потенциала на срезе разрядного канала, приводящий к появлению выходящего из канала ускоренного потока ионов.

\section{Введение}

К настоящему моменту в мире накопился довольно обширный объем научного материала по разряду постоянного тока, помещенному в магнитное поле с преимущественной радиальной компонентой. Помимо того, что уже в течение нескольких десятилетий такой разряд является объектом пристального фундаментального исследования, он также находит свое практическое применение в качестве рабочего процесса электрореактивных холловских двигателей [1,2]. Несмотря на более чем сороколетнюю историю практического применения разряда, целый ряд фундаментальных вопросов и сегодня являются предметом активного исследования и дискуссионого обсуждения [3]. Это, прежде всего, механизмы переноса тока от катода к аноду поперек магнитного поля и физические причины многочисленных колебаний, возникающих в разряде $[1,4]$.

Основной физической идеей, заложенной в рабочий процесс холловского двигателя, является организация азимутального дрейфа электронов в скрещенных продольном электрическом и радиальном магнитном полях [3]. Электрическое поле в канале вблизи его среза возникает благодаря прикатодному падению потенциала. Плазма в канале поддерживается за счет ионизации газа электронами, эмитируемыми с катода. В соответствии с современной картиной физических процессов, происходящих в разряде, значительный вклад в перенос электронов от катода к аноду поперек магнитного поля дают не только столкновения электронов с другими частицами плазмы, но и процессы, связанные с пристеночной проводимостью [1], возникновением дрейфовой неустойчивости и генерацией вторичных электронов при соударении электронов и ионов со стенками канала [4-6].
Известно, что в емкостном высокочастотном (ВЧ) разряде вблизи электродов формируются слои пространственного заряда, где возникают квазистационарные электрические поля [7]. Можно предположить, что наличие внешнего радиального магнитного поля приведет к появлению азимутального дрейфа электронов в областях локализации слоев объемного заряда. В то же время ВЧ разряд не требует использования контактирующих c плазмой металлических электродов и не нуждается в эмитирующем катоде для своего поддержания. Это снимает трудности, связанные с корректным описанием электронного транспорта в разрядном канале, и устраняет проблему переосаждения металла из-за распыления электродов. Указанные соображения дают основание для исследований возможности использования емкостного ВЧ разряда с радиальным внешним магнитным полем как рабочего процесса в электрореактивных двигателях и источниках ионов. В настоящей работе поставленная задача выполнена с помошью математического моделирования.

\section{Методика расчетов}

Численное моделирование физических процессов в емкостном ВЧ разряде, помещенном во внешнее радиальное магнитное поле, выполнялось посредством двумерной осесимметричной нерелятивистской версии кода KAPAT [8], в котором решаются нестационарные уравнения Максвелла с различными материальными уравнениями. В настоящей работе плазма моделируется методом крупных частиц (РІС-метод) с учетом всех компонент скоростей частиц. Условия применимости счетной схемы имеют следующий вид: шаг сетки значительно меньше минимального размера любого моделируемого 

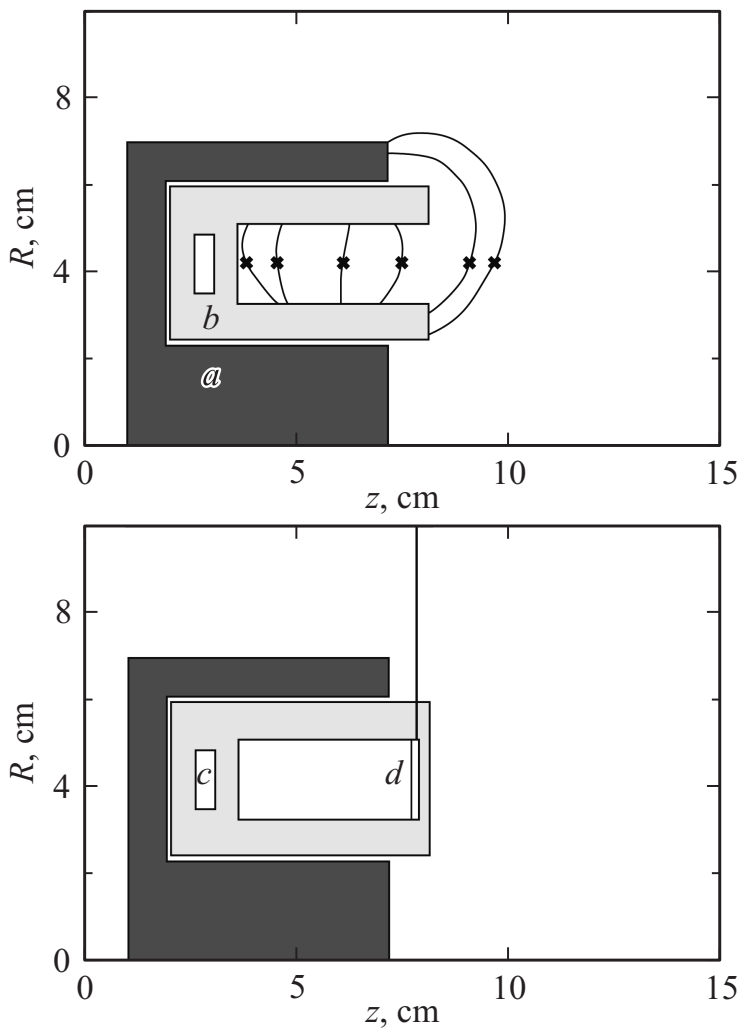

Рис. 1. Базовая геометрия моделируемого промежутка с силовыми линиями магнитного поля, проходящими через отмеченные крестом точки (верх.) и расположение электродов в случае закрытого канала (нижн.). Буквами обозначены: $a-$ проводящий корпус, $b-$ диэлектрический канал, $c-$ активный электрод, $d-$ заземленный электрод.

геометрического объекта, а также дебаевской длины плазмы; шаг по времени значительно меньше любого характерного времени системы, в том числе периода ленгмюровских колебаний, периода вращения в магнитном поле, времени свободного пробега частиц; внутри ячейки и внутри дебаевской сферы имеется несколько десятков РіС-частиц. В представляемых результатах эти требования соблюдены. За время с момента написания кода [8] были проведены все возможные сравнения с аналитическими, экспериментальными результатами, сравнение с численными результатами других групп.

Счетная область представляла собой цилиндр длиной $15 \mathrm{~cm}$ и радиусом $10 \mathrm{~cm}$. Использовавшаяся геометрия источника плазмы близка к геомерии холловского плазменного двигателя [1] (рис. 1). В настоящей работе рассмотрено 3 случая. В первом случае разрядный канал с торцов был закрыт электродами. К левому электроду прикладывалось ВЧ напряжение, правый электрод был заземлен. Во втором случае правый электрод параллельным переносом был вынесен за пределы канала. В третьем случае правый электрод был смещен к границе счетной области так, что его поверхность не перекрывала выходное отверстие канала. Рассчитывалось поле тороидального активно- го электрода, на который подавалось гармоническое ВЧ напряжение с частотой $12.5 \mathrm{MHz}$ и амплитудой в диапазоне $0.5-2 \mathrm{kV}$. В начальный момент в счетной области задавалась плотность нейтральных атомов ксенона с постоянной концентрацией внутри канала и на порядок меньшей концентрацией вне канала, а также начальная плазма, состоящая из ионов и электронов с концентрацией $10^{10} \mathrm{~cm}^{-3}$. В процессе численного моделирования рассматривалась динамика заряженных частиц плазмы в приложенных и собственных самосогласованных электромагнитных полях. При столкновении электронов с поверхностями заземленного электрода, диэлектрического канала моделировалась вторичная эмиссия электронов, причем использовалась простейшая модель с постоянным коэффициентом вторичной ионно-электронной эмиссии $\gamma$, равным 0.1. В объеме моделировались упругие и ионизирующие столкновения электронов с атомами ксенона, концентрация которых задавалась в диапазоне $3 \cdot 10^{13}-3 \cdot 10^{14} \mathrm{~cm}^{-3}$. Соответствующие сечения столкновений были взяты из работы [9]. Образовавшиеся при ионизации электрон-ионные пары включались в последующее моделирование, увеличивая плотность плазмы. С заземленного электрода эмитировался ток $0.01,0.1,1 \mathrm{~A}$, соответствующий току термоэмиссии. Индукция внешнего магнитного поля лежала в диапазоне 50-200 Gs. Для уменьшения времени счета масса ионов $M$ взята равной 2000 массам электронов $m$.

Как правило, просчитываемое время эволюции системы составляло $8 \mu \mathrm{s}$. В результаты моделирования записывались итоговые параметры численной системы в том случае, если средняя за период колебаний ВЧ поля концентрация плазмы в разрядном канале за моделируемый промежуток времени выходила на стационарное значение. Когда этого не происходило, концентрация либо спадала к нулю, т.е. начальная плазма рассеивалась, и считалось, что разряд в этих условиях гаснет, либо концентрация достигала величины порядка $10^{12} \mathrm{~cm}^{-3}$, и компьютерное время, требуемое для обсчета подобной системы в рамках выбранной нами численной модели, становилось слишком большим (из-за необходимости уменьшать шаг сетки и временной шаг).

Вышеописанная численная модель была протестирована для тлеющего разряда постоянного тока в циллиндрическом сосуде с плоскими электродами на его торцах и для емкостного ВЧ разряда в той же геометрии (частота напряжения на электродах - $12.5 \mathrm{MHz}$ ), после чего было произведено сравнение результатов с полученными ранее экспериментальными данными. В итоге моделирование корректно воспроизвело качественную структуру как приэлектродных слоев, так и центральных областей плазмы ВЧ разряда и разряда постоянного тока. Однако концентрация ионов во всем разряде оказалась заниженной в 1.5 раза относительно эксперимента, что было вызвано искусственным уменьшением массы ионов до 2000 масс электронов. В тех случаях, когда подобного уменьшения не делалось, концентрация примерно соответствовала экспериментальной (с точностью 
до $10 \%$ от ее значения). При этом значение коэффициента вторичной ионно-электронной эмиссии $\gamma$, равное 0.1 , было подобрано для наилучшегно согласования данных моделирования и эксперимента.

\section{Результаты расчетов}

Численное моделирование показало, что в присутствии радиального магнитного поля основные свойства емкостного ВЧ разряда сохраняются. На рис. 2 помещено мгновенное распределение потенциала плазмы по длине канала. Как видно, оно имеет типичный для емкостного ВЧ разряда вид: рядом с электродами формруются слои пространственного заряда, где происходит существенное падение потенциала, в центре канала существует область с практически постоянным потенциалом.

На рис. 3 показана временна́я зависимость компонент ВЧ электрического поля вблизи активного и заземленного электродов. Рисунок иллюстрирует тот факт, что рядом с электродами продольное ВЧ электрическое поле, во-первых, изменяется не гармонично, а, во-вторых, имеет постоянную по времени составляющую, обеспечивающую существование квазистационарного падения потенциала вблизи электродов.

На рис. 4,5 показаны распределения концентрации и кинетической энергии электронов и ионов вдоль оси канала. Можно заметить, что плазма формируется в центральной части канала, где продольное падение потенциала незначительно. В области приэлектродных слоев пространственного заряда средняя энергия электронов существенно выше, чем в центральной области. Наибольшей энергией вблизи активного электрода обладают электроны, родившиеся на нем в результате ионноэлектронной эмиссии и ускоренные приэлектродными полями в направлении плазмы. Вблизи заземленного электрода также велика кинетическая энергия эмитированных с него электронов. Ионы же, в свою очередь, ускоряются квазистационарными полями в направлении от плазмы к электродам. Это дает возможность получить на выходе из открытого канала поток ускоренных ионов.

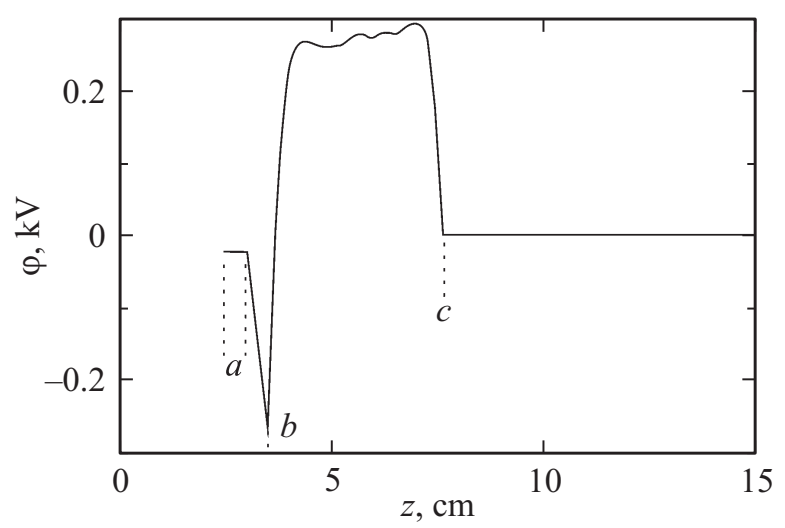

Рис. 2. Мгновенное распределение потенциала в закрытом разрядном канале $(R=4.25 \mathrm{~cm})$.
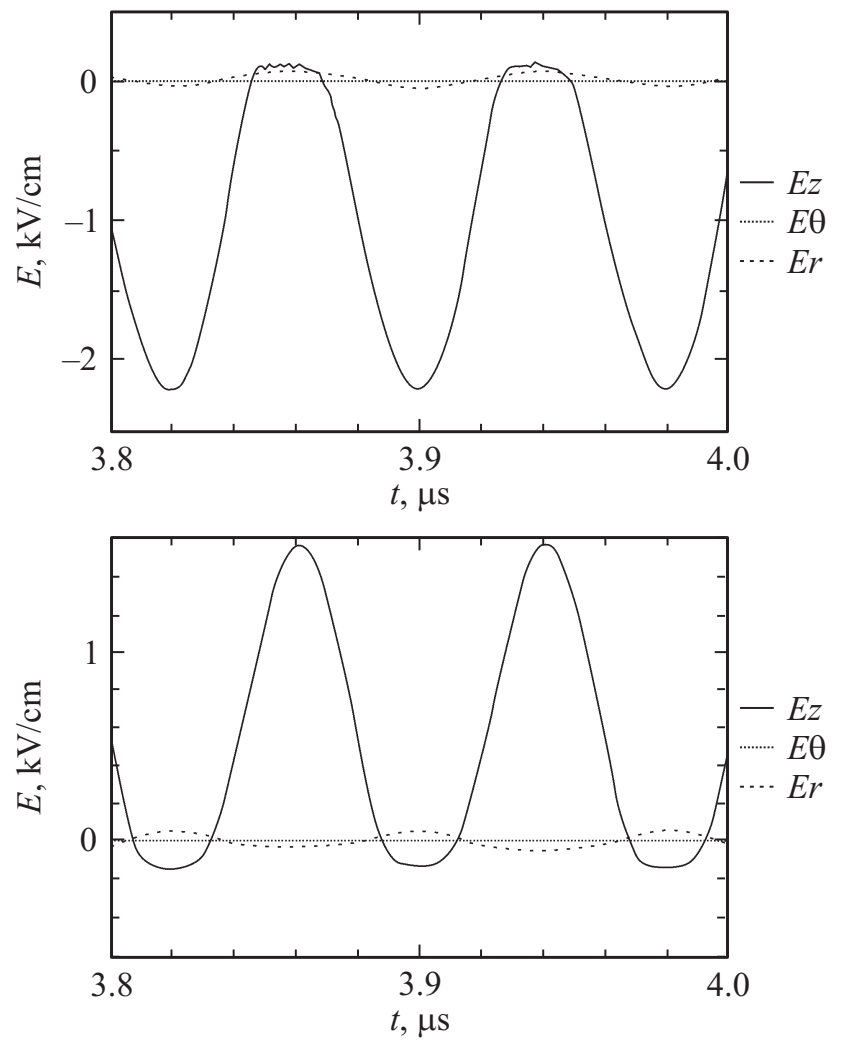

Рис. 3. Временна́я зависимость компонент электрического поля вблизи активного (верхний график) и заземленного (нижний график) электродов.

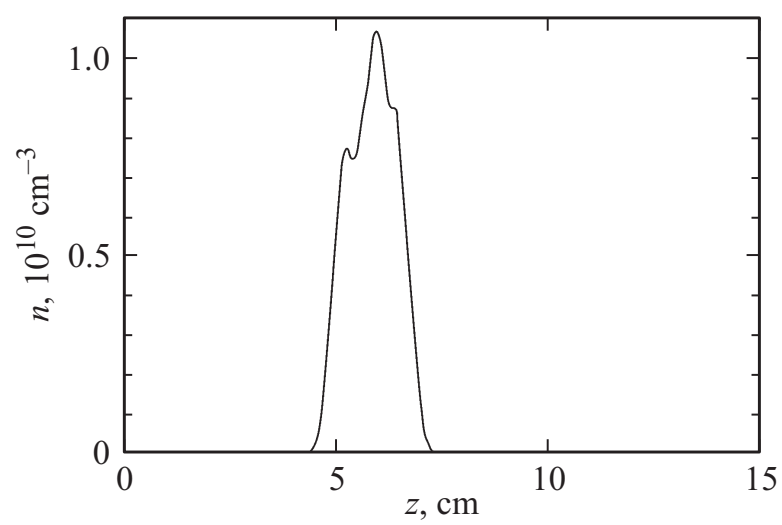

Рис. 4. Распределение плотности ионов вдоль разрядного канала.

Наличие радиального магнитного поля проявляется в возникновении азимутального дрейфа электронов в приэлектродных областях (рис. 6). Необходимо отметить, что электроны, эмитированные с поверхности заземленного электрода и дрейфующие по азимуту в скерщенных $\mathbf{E} \times \mathbf{B}$ полях, локализуются вблизи этого электрода, так как их продвижение в глубь плазмы ограничено радиальным магнитным полем. В геометрии разряда, когда правый электрод вынесен из канала, эмитированные электроны не попадают в канал, так как 

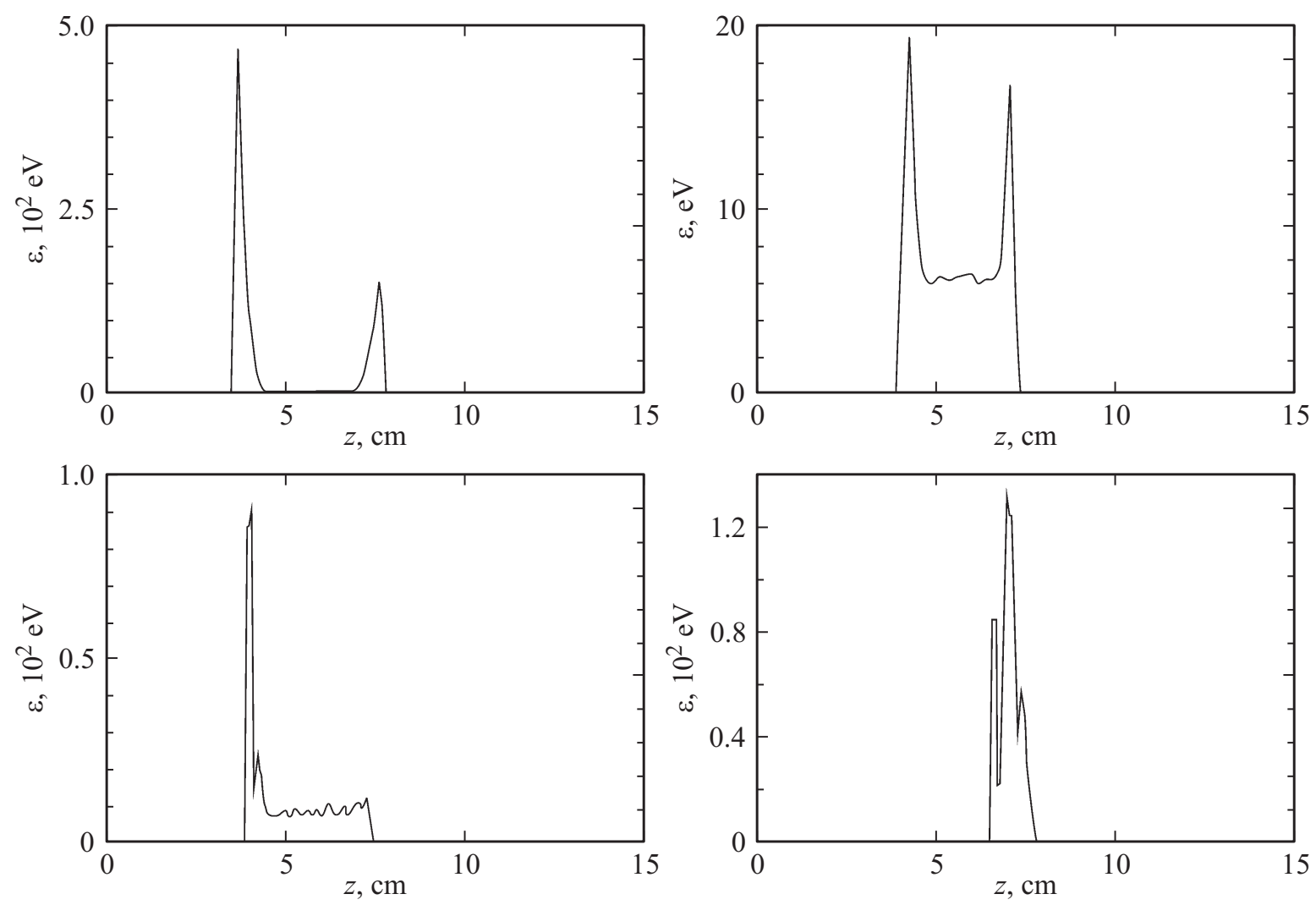

Рис. 5. Распределение вдоль канала кинетической энергии частиц различных сортов: ионов (лев. верх.), электронов, рожденных посредством ионизации нейтрального газа (прав. верх.), электронов, рожденных в процессах ионно-электронной эмиссии в области активного электрода (лес. ниж.), электронов, эмитированных с заземленного электрода (прав. ниж.).

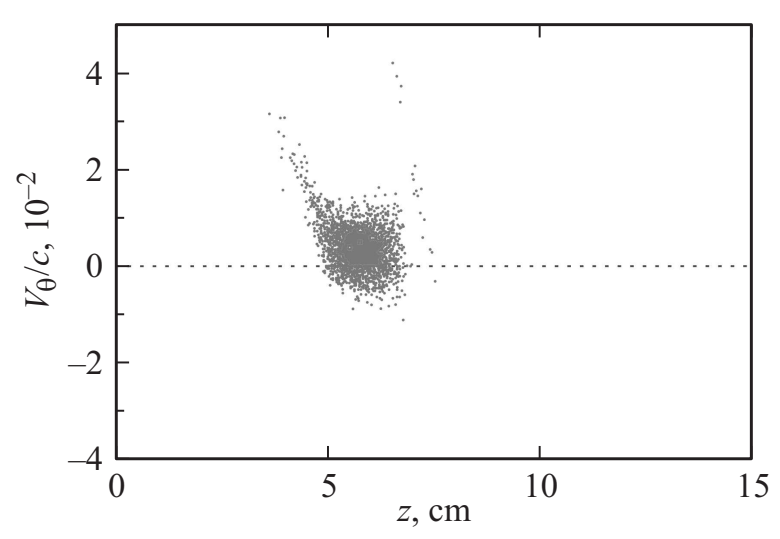

Рис. 6. Мгновенное распределение азимутальной скорости электронов (крупных частиц) вдоль разрядного канала.

они удерживаются радиальным магнитным полем вне канала (рис. 7).

Необходимо подчеркнуть, что для поддержания емкостного ВЧ разряда, помещенного во внешнее магнитное поле, наличие эмиссии с одного из электродов не является обязательным. Разряд поддерживается за счет диссипации энергии ВЧ полей в результате как бесстолкновительного, так и столкновительного механизмов [7]. В целом рост эмиссии электронов с заземленного электрода сопровождается увеличением плотности плазмы

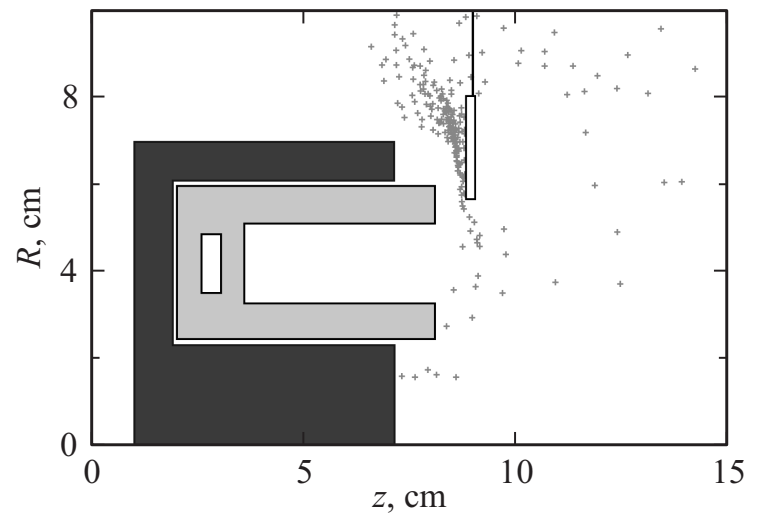

Рис. 7. Локализация электронов, эмитированных с заземленного электрода в случае открытого канала.

и сужением приэлектродных слоев пространственного заряда. Однако одновременно рост тока эмиссии вызывает падение приэлектродного потенциала у заземленного электрода, что связано с частичной компенсацией объемного заряда положительных ионов эмитированными электронами.

Расчеты показали, что увеличение индукции внешнего магнитного поля сопровождается ростом плотности плазмы, однако энергия ионов при этом уменьшается (рис. 8). Рост концентрации атомов и амплитуды ВЧ 

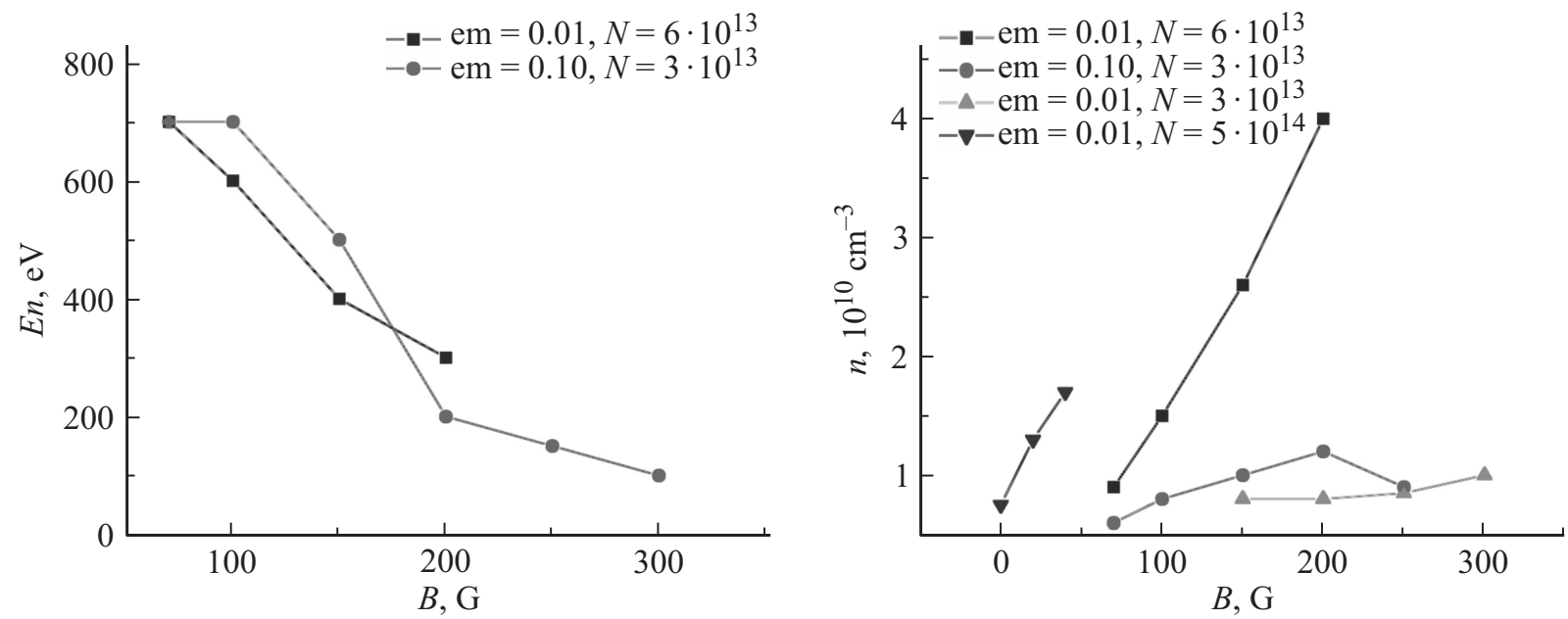

Рис. 8. Зависимость энергии уходящих к активному электроду ионов (лев.) и концентрации плазмы (прав.) от индукции внешнего магнитного поля. ет - ток эмиссии с заземленного электрода (в А), $N$ - концентрация нейтрального газа в разрядном канале $\left(\right.$ в $\left.\mathrm{cm}^{-3}\right)$.

напряжения, приложенного к электродам, приводит к росту плотности плазмы. Увеличение амплитуды ВЧ напряжения приводит к возрастанию энергии ионов на выходе из разрядного канала.

Частично вышеописанные результаты моделирования были экспериментально проверены в выполненной позднее работе [10], где исследовался ионный поток, полученный при помощи емкостного ВЧ разряда в радиальном магнитном поле. Во всем исследованном в работе [10] диапазоне разрядных мощностей амплитуда ВЧ напряжения практически не менялась и составляла величину порядка $450 \mathrm{~V}$, что существенно расходится с численным моделированием, в котором концентрация начальной плазмы при значениях амплитуды напряжения на активном электроде, меньших $700 \mathrm{~V}$, не выходила за стационарное значение и равномерно спадала к нулю, т.е. моделируемый разряд гас. Однако при этом все зависимости параметров ионного потока (его плотности, средней энергии) от внешних параметров разряда в моделировании и в эксперименте качественно оказались одинаковыми. В частности, в обоих случаях средняя энергия выходящих из разрядного канала ионов была приблизительно равна половине амплитуды разрядного напряжения, а плотность ионного потока росла с ростом величины магнитного поля на срезе канала.

\section{Заключение}

Расчеты, выполненные в настоящей работе, показали, что вблизи электродов емкостного ВЧ разряда, помещенного в радиальное внешнее магнитное поле, возникают приэлектродные скачки потенциала, причем скачок потенциала у заземленного электрода возрастает с уменьшением тока эмиссии. Наличие продольного электрического поля и радиального магнитного поля приводит к возникновению азимутального дрейфа электронов. Рост напряжения, приложенного к электродам, тока эмиссии, плотности атомов и величины магнитного поля вызывают увеличение плотности электронов в канале. Скачок потенциала, усредненного за период колебаний электрического поля, возникающий вблизи среза канала, является ускоряющим для ионов, покидающих разрядный канал, что выражается в формировании потока ионов на выходе из источника плазмы.

Работа выполнена при поддержке гранта РФФИ № 1302-01089a.

\section{Список литературы}

[1] Морозов А.И. Введение в плазмодинамику. 2-е изд. М.: Физматлит, 2008. $576 \mathrm{c.}$

[2] Горшков О.А., Муравлев В.А., Шагайда А.А. Холловские и ионные плазменные двигатели для космических аппаратов. М.: Машиностроение, 2008. 280 с.

[3] Ким В.П. // ЖТФ. 2015. Т. 85. Вып. 3. С. 45-59.

[4] Бугрова А.И., Харчевников В.К., Якунин С.А. // Теплофизика высоких температур. 1981. Т. 19. № 5. С. 1045-1049.

[5] Coche P., Garrigues L. Paper IEPC-2011-255 at the $32^{\text {nd }}$ International Electric Propulsion Conf. Germany. Wiesbaden, 2011.

[6] Tomilin D.A., Gorshkov O.A. Paper IEPC-2011-023 at the $32^{\text {nd }}$ International Electric Propulsion Conf. Germany. Wiesbaden, 2011.

[7] Райзер Ю.П., Шнейдер М.Н., Яценко Н.А. Высокочастотный емкостный разряд: Физика. Техника эксперимента. Приложения. М.: Изд-во МФТИ, 1995. 320 с.

[8] Tarakanov V.P. User's Manual for Code KARAT (Berkeley Research Associates, Springfield, VA, 1992). Тараканов В.П. 3D электродинамический код „Карат“. Дис. РАН. Объединенный институт высоких температур.

[9] de Heer F.J., Jansen R.H., van der Kaay W. // J. Phys. B. Atom. Molec. Phys. 1979. Vol. 12, N 6. P. 979-1002.

[10] Задириев И.И., Рухадзе А.А., Кралькина Е.А. и др. // Прикладная физика. 2015. № 6. С. 57-60. 